\title{
ARTICLE
}

\section{Methods and procedures for shielding analyses for the SNS}

\author{
Irina Popova*, Franz X. Gallmeier, Erik B. Iverson, Wei Lu and Igor Remec
}

\begin{abstract}
Oak Ridge National Laboratory, Spallation Neutron Source, PO Box 2008, MS6475, Oak Ridge, TN 37831-6471, U.S.A
\end{abstract}
\begin{abstract}
In order to provide radiologically safe Spallation Neutron Source operation, shielding analyses are performed according to Oak Ridge National Laboratory internal regulations and to comply with the Code of Federal Regulations. An overview of on-going shielding work for the accelerator facility and neutrons beam lines, methods used for the analyses, and associated procedures and regulations are presented. Methods used to perform shielding analyses are described as well.
\end{abstract}

\section{Keywords: high-energy accelerator; Monte Carlo; shielding design; radiation protection}

\section{Introduction}

The Spallation Neutron Source (SNS) in Oak Ridge, Tennessee, is an accelerator driven neutron scattering facility for materials research. Presently SNS operates at 1 megawatt (MW) proton beam power incident on a mercury target with a proton beam energy of $1 \mathrm{GeV}$ and $60 \mathrm{~Hz}$ repetition rate. The facility is still ramping up the power to reach the designed $1.4 \mathrm{MW}$ on target. SNS consists of accelerator system, target system, and a world-class suite of neutron scattering instruments to benefit a user program of material, life-science and fundamental physics research.

The SNS accelerator is powered by an $\mathrm{H}$ - beam, which transfers after acceleration into proton beam and consists of the linear accelerator (LINAC), the high-energy-beam-transfer line (HEBT), the accumulator ring and the ring-to-target-beam-transfer line (RTBT). The high energy neutrons resulting from the proton initiated spallation reactions in the mercury target are converted to thermal and cold neutrons by one ambient water and three supercritical hydrogen moderators placed above and below the target. The thermalized neutrons are directed to the neutron scattering instruments through neutron beam lines. There are 18 beam lines, 6 of which serve two instruments each, so the facility is able to accommodate 24 instruments.

All stages of the SNS development required and still require significant research and development in the field of radiological shielding design to assure safety from a radiation-protection point of view for accelerator, target system, and scattering instruments. At present, most of the shielding work is concentrated on the instrument beam lines and their enclosures in order to commission and provide safe operation in the future.

*Corresponding author. Email: popovai@ornl.gov
However, there is still support provided for the accelerator facility to redesign parts of the accelerator structures, to design storage containers for removed activated components and test stands for accelerator structures, and radiation protection analyses.

\section{Overview on procedures, regulations and facility stages}

Shielding analyses for SNS facilities, both accelerator and target with scattering beam lines, are performed to meet the requirements of SNS-OPM 2.H-5 "SNS Radiation Safety Policy" and Section 7.8 of the "Spallation Neutron Source Final Safety Assessment Document for Neutron Facilities," and to comply with 10 CFR 865 regulations.

According to the regulations, the design dose rate in generally occupied area is below $0.25 \mathrm{mrem} / \mathrm{h}$ at $30 \mathrm{~cm}$ from the shielding surface and further away. Historically there were multiple stages of shielding design:

- Before and during the accelerator and target facility construction, the initial design;

- Support of the accelerator and later the target commissioning;

- $\quad$ Shielding support during the power ramp up and operation, which is on-going.

\subsection{Initial design}

The SNS operates a high current and high power accelerator. The accelerator systems are designed to be maintained hands on. This sets the acceptable beam losses to the level of around 1 Watt per meter limiting the activation level in the accelerator and proton beam line tunnels. This also determines the bulk shielding requirement for the accelerator tunnels resulting in about five meters of soil on top of the tunnels to ensure dose 
rates below $0.25 \mathrm{mrem} / \mathrm{hr}$.

The target monolith housing around the target station has the task to provide shielding to a dose rate of 0.25 $\mathrm{mrem} / \mathrm{hr}$, both for personnel protection and neutron background reasons. Low-neutron background is desired by the neutron scattering instruments. The SNS design includes 18 main shutters, which allow to close each neutron beam lines individually while SNS is operating, to enable access to the instrument cave. Another important piece of shielding is the target cart, a $5 \mathrm{~m}$ long steel plug providing shielding downstream of the target, which is mounted on its front.

\subsection{Commissioning}

Commissioning of the accelerator system is a critical step in the transition from the fabrication and installation phase to the operations phase. Detailed predictions for radiation fields induced inside and outside of the accelerator tunnel were calculated for all commissioning steps, according to the SNS Commissioning Program Plan [1]. On the basis of neutronics analyses, proper shielding was developed and installed in key locations to reduce dose rates in occupied areas.

The beam power deposited locally in the accelerator tunnel during the commissioning phases greatly exceeded typical operational line losses, which are on the order of $1 \mathrm{~W} /$ meter, with the consequence of very high radiation fields. Proper temporary shielding was installed in local areas near beam termination points (beam stops and beam collectors) and some critical locations, such as penetrations, in order to minimize dose rates in normally occupied areas.

Each commissioning step was preceded by an Accelerator Readiness Review (ARR). The ARR process verifies the machine readiness for each commissioning step. ARRs are conducted in accordance with the requirements established in DOE Order 5480.25, "Safety of Accelerator Facilities."

\subsection{Power ramp up and operation}

During power ramp up, shielding analyses are mainly concentrated on neutron scattering beam lines and instrument enclosure shielding design. Neutron beams are contaminated by a large fraction of fast neutrons with energies up to the driving proton energy. The fast neutrons can be attenuated by choppers making use of the pulsed beam structure, the discrimination of fast and thermal neutrons by time of flight, and by curved neutron guides. In either way the neutron flight paths have to be packed into heavy thick shielding that needs to be custom tailored to each specific instrument including the neutron guides, choppers, sample environments, detectors and beam stops. Before allowing beam to an instrument by opening the beamline's primary shutter, the instrument team has to defend the instrument design at an Instrument Readiness Review (IRR) and proof that it can be operated safely. The IRR ascertains that the instrument has been designed, constructed, and installed to allow safe operation and maintenance for both staff and general users. The IRR is conducted by the SNS Instrument Safety Committee (ISSC) and is expanded as necessary at the direction of the ISSC chairman. The IRR committee gives recommendations to the operation manager for authorization of the operation of instruments. The ISSC performs an independent evaluation of instruments as they are constructed, commissioned, operated, and modified.

Guidelines for the SNS neutron beam line shielding calculations [2] sets standards for the beam line and instrument enclosure analyses and helps to prepare for the Instrument Readiness Review (IRR).

Additionally to the beam lines and the instrument enclosure shielding design there is still a need for radiation protection and shielding analyses for the accelerator facility as well such as

- Redesign of some parts of the facility and facility upgrades;

- Design of additional structures, mostly for testing purposes;

- Design of storage/transport containers for removed irradiated structures from the accelerator tunnel;

- Radiation-protection analyses.

Any change in the radiation shielding before being made on the site is going through the Radiation Safety Committee (RSC).

\section{Methods and codes}

Radiation transport calculations for shielding design and radiation protection analyses are performed mainly with the Monte Carlo code MCNPX version 2.5.0 [3] with realistic three-dimensional geometric description for all facility components and support structures. The MCNPX code simulates the particle transport of hadrons, continuous energy loss of charged particles in matter, elastic and nonelastic hadron interactions, secondary particle generation (here mainly gamma ray and neutrons) and their transport. Geometry splitting is applied to force particles towards the outside of the shielding for deep penetration calculations in order. MCNPX calculations are usually running on multiprocessors computers in the parallel mode.

Effective dose rates are obtained by folding neutron and gamma ray fluxes with flux-to-dose conversion coefficients, which are taken from standardized for SNS neutron and gamma ray flux-to-dose conversion factors libraries [4].

Analyses for residual dose calculations for the parts of facility, and for developing storage/transport containers for extracted irradiated structures are performed in three steps. In the first step, reaction rates in the requested structures were calculated using MCNPX. In the second step, isotope production rates are fed into the Activation Script [5]. This script provides the interface between MCNPX and the transmutation codes CINDER'90, ORIHET3 and SP-FISPACT. CINDER'90 is usually applied to obtain the time 
dependence of the isotope buildup and decay for given locations according to the provided operational scenario. From the transmutation code outputs, gamma ray decay spectra and gammas ray power are extracted.

In the third step, the extracted gamma spectra are formatted into source descriptions for MCNPX to perform decay gamma ray transport calculations. For simple geometry problems, like residual dose rate estimation near accelerator pipe, photon source spectra are converted to dose rates manually by dividing by the area corresponding to the distance from the beam pipe and folding with flux to dose conversion factors.

For the radionuclide inventory analyses, steps one and two are applied, and then isotope concentrations are extracted from the transmutation code outputs for structural accelerator materials, for water and soil.

\section{Current shielding analyses}

Presently shielding work is mostly concentrated on neutron beam line shielding. Neutron beam lines require bulk shielding because of a large high-energy component in the neutron beam streaming through the guide to the instruments.

\subsection{Beam lines shielding}

This section illustrates in couple example the complexity of the beam line shielding analyses for the instruments. Beam line shielding analyses are logically divided into two sets:

- Analysis of the incident beam line;

- Analysis of the instrument cave or enclosure, including the neutron beam stop.

Neutron beam lines at the SNS can be straight (allowing passage of fast and high-energy neutrons) or curved (relying on neutron optics to transport thermal and cold neutrons). All beam lines have primary shutters within the shielding monolith. Many beam lines also include secondary shutters, either to allow multiple instruments to use a single primary shutter or to permit more rapid closure of the beam and faster personnel access to the instrument sample area. The shielding analyst's prime task is to design bulk shielding around the beam line. Any beam obstructions expected to affect the shielding such as secondary shutters, slits, beam choppers and slits are taken into consideration.

Beam line and instrument shielding analyses are

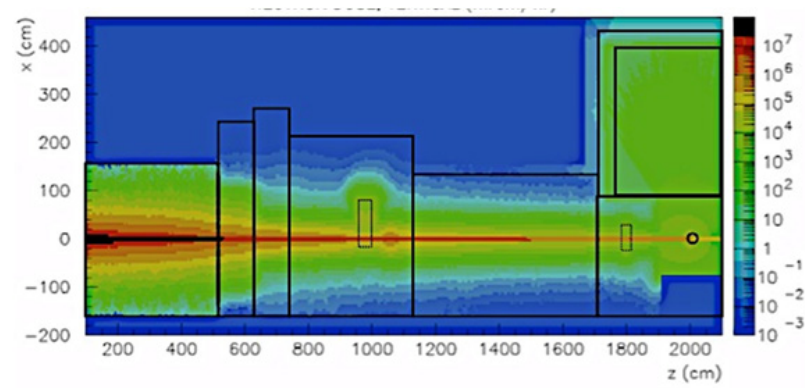

Figure 1. Dose rate map in elevation view of SEQOUIA beam line, $\mathrm{mrem} / \mathrm{h}$. performed using source terms describing the neutron in-scattering into the beam lines starting at about 1 meter distance from the moderator faces.

Figure 1 shows an example of the dose rates along the flight path for beam line 17, the SEQOUIA instrument. This beam line is straight and its sample is positioned $20 \mathrm{~m}$ from the moderator. Black lines represent the beam line geometry on the all figures. Dotted lines represent cavities for the choppers. The beam line model starts at $100 \mathrm{~cm}$ from the moderator and extends to $1709 \mathrm{~cm}$ from moderator. Lines after $1709 \mathrm{~cm}$ from the moderator represent the front portion of the instrument enclosure shielding.

The instrument enclosure shielding analyses will include two separate analyses: the beam stop and the enclosure shielding design. Enclosure shielding is designed for so called "normal operation" beam conditions (beam with limited energy bandwidth) in case an area monitor activates the closing of the shutter in case of elevated dose rates. Otherwise accident-case beam conditions are considered such as a white unobstructed beam runs into the piece of equipment or the worst case samples. Both polyethylene and steel samples are used for those analyses. The beam stop shielding must be designed for white unobstructed beam with no sample inserted.

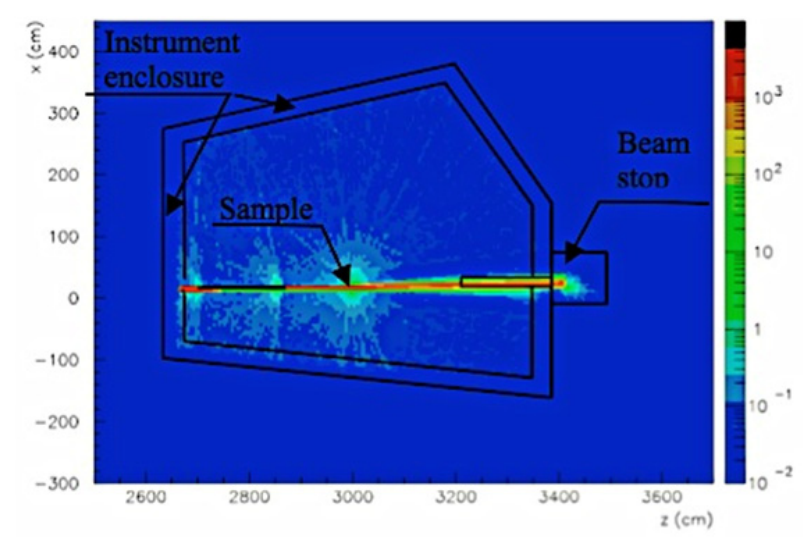

Figure 2. Dose rate map in horizontal view of the MANDI instrument enclosure, $\mathrm{mrem} / \mathrm{h}$.

Figure 2 represents the dose rates inside and outside of the MANDI instrument enclosure at beam line $11 \mathrm{~b}$. It is an example of a curved beam line, and with a relatively small guide opening of $7 \mathrm{~mm}$ by $7 \mathrm{~mm}$ at the end of the guide and long flight path. The sample position is located at $28.6 \mathrm{~m}$ from the moderator. Analyses for the shielding enclosure were performed in two steps. At first, a boundary source on the inner surface of the enclosure entrance was collected. Its surface is located $26.7 \mathrm{~m}$ from the moderator. Secondly, particles from the boundary source were propagated into the enclosure and followed thru the shielding wall to the outside of the enclosure.

Figure 3 shows a dose rate map for the beam line 16b shielding enclosure, which is the VISION instrument. It is an example of a straight beam line with the sample at $16 \mathrm{~m}$ from the moderator and a beam stop integrated into 
the enclosure.

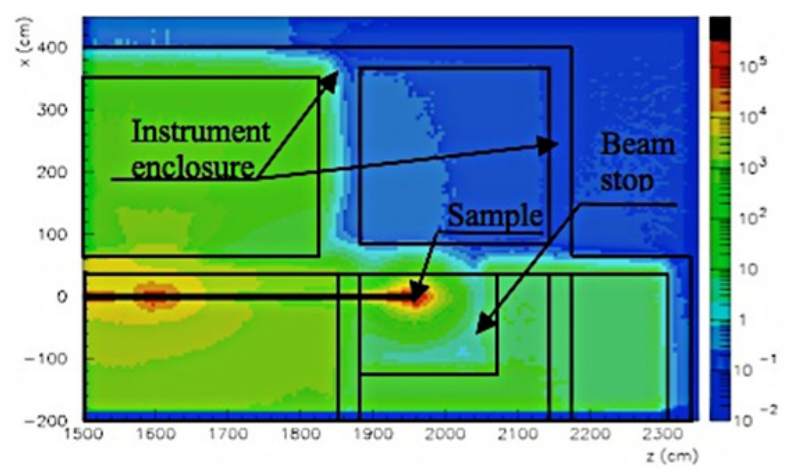

Figure 3. Dose rate map in horizontal view of the VISION instrument, the neutron beam terminates in the beam stop, $\mathrm{mrem} / \mathrm{h}$

\subsection{Shielding analyses for accelerator facility}

Example of radiation protection analyses is summarizing the response of the area radiation monitors ("chipmunks") to the maximum possible accidental beam spill around the accelerator facility and to evaluate whether any beam-spill accidents would be detected by at least two chipmunks.

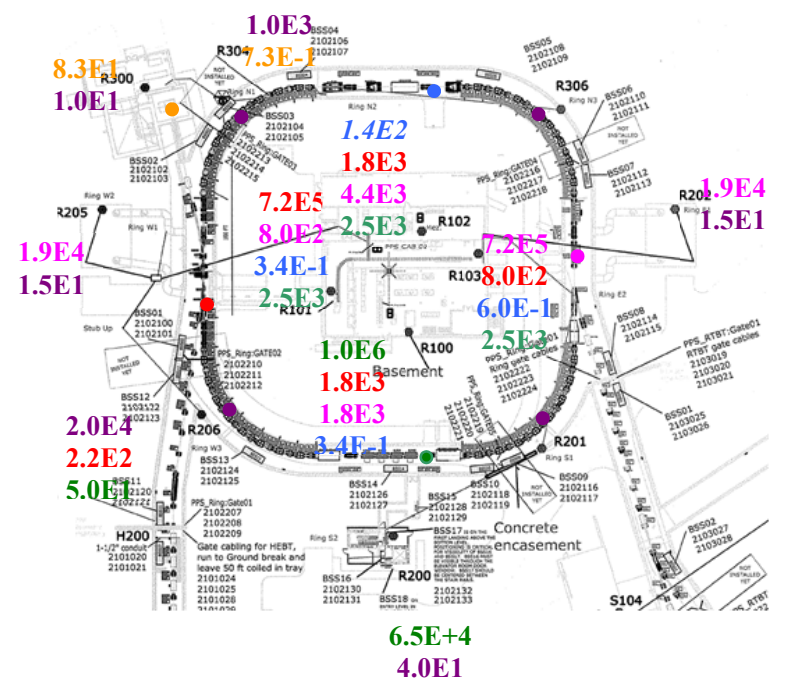

Figure 4. Dose rates at chipmunks in the storage ring, $\mathrm{mrem} / \mathrm{h}$.

As an example, Figure 4 summarizes chipmunk readings in case of a possible maximum beam spill in the ring section of accelerator. The colored dot shows the location of the beam spill at a thick target or the center of the beam spill on the beam pipe assuming a Gaussian distribution. The colored numbers near each chipmunk shows the dose rate at them. The color of the number refers to the dose rate measured by the chipmunk when the spill appears in the place marked with the same color. Chipmunk locations are marked by the letter R. Analyses show that the existing chipmunk locations are satisfactory to measure any elevated dose rate from accident conditions in the accelerator.

One of the most demanding tasks is design of storage containers. According to the accelerator operations plan some components are replaced because they have reached their end-of-life or because of design improvements. These components must be safely removed, placed in a container for storage, and moved from the accelerator building. Figure 5 shows an example of storage container design for the HEBT momentum dump. The blue lines represent the container, which is cylindrical in shape and surrounds the beam stop. The container profile changes thickness along its length to match the beam dump residual activation. The container was developed with the criteria that the dose rate outside the container after a one year cool down will not exceed $5 \mathrm{mrem} / \mathrm{h}$ at $30 \mathrm{~cm}$ from the container surface.

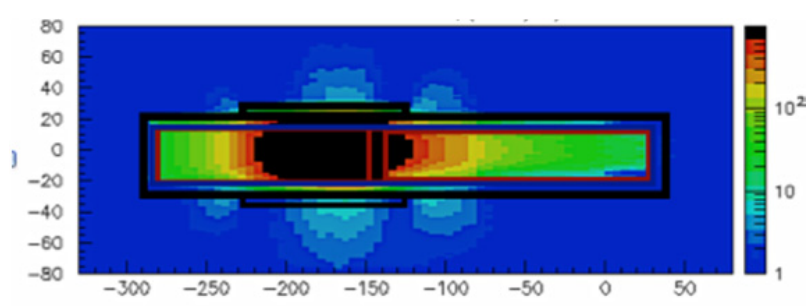

Figure 5. Dose rates map inside and outside HEBT momentum dump container.

\section{Conclusion}

According to the regulations, all the shielding is designed to meet $0.25 \mathrm{mrem} / \mathrm{h}$ and lower dose at $30 \mathrm{~cm}$ distances from the shielding surface and above. Presently the most of the shielding efforts are concentrated on neutron beam line shielding design in order to bring instruments online. There are established procedures that guide neutron beam line shielding design. There is still undergoing shielding work to support accelerator facility.

\section{Acknowledgements}

Work supported by the Division of Materials Science, U.S. Department of Energy, under contract number DE-AC05-96OR22464 with UT-Battelle Corporation for ORNL.

\section{References}

[1] Spallation neutron source commissioning program plan, Oak Ridge National Laboratory, SNS 100000000P-PN0004-R00, July 2002, Phys. Rev. Lett. 25 (1997).

[2] I. Popova, Guidelines for Shielding Calculations for SNS Neutron Beam Lines, Oak Ridge National Laboratory, SNS 106100299-WI0001-R00.

[3] Pelowitz D. B., editor, MCNPX User's Manual Version 2.5.0, LA-CP-05-0369, Los Alamos National Laboratory, Los Alamos, April 2005.

[4] I. Popova, Flux to Dose Conversion Factors, SNS-NFDD-NSD-TR-0001, R00, October 2009.

[5] CINDER Version 1.05: Code system for actinide transmutation calculations, RSICC C00755 PC586 00, September 2009. 